\title{
Widely tunable microwave photonic notch filter based on slow and fast light effects
}

\author{
Xue, Weiqi; Sales, Salvador; Mørk, Jesper; Capmany, Jose
}

Published in:

IEEE Photonics Technology Letters

Link to article, DOI:

10.1109/LPT.2008.2009468

Publication date:

2009

Document Version

Publisher's PDF, also known as Version of record

Link back to DTU Orbit

Citation (APA):

Xue, W., Sales, S., Mørk, J., \& Capmany, J. (2009). Widely tunable microwave photonic notch filter based on slow and fast light effects. IEEE Photonics Technology Letters, 21(3), 167-169.

https://doi.org/10.1109/LPT.2008.2009468

\section{General rights}

Copyright and moral rights for the publications made accessible in the public portal are retained by the authors and/or other copyright owners and it is a condition of accessing publications that users recognise and abide by the legal requirements associated with these rights.

- Users may download and print one copy of any publication from the public portal for the purpose of private study or research.

- You may not further distribute the material or use it for any profit-making activity or commercial gain

- You may freely distribute the URL identifying the publication in the public portal 


\title{
Widely Tunable Microwave Photonic Notch Filter Based on Slow and Fast Light Effects
}

\author{
Weiqi Xue, Salvador Sales, Senior Member, IEEE, Jesper Mørk, and José Capmany, Fellow, IEEE
}

\begin{abstract}
A continuously tunable microwave photonic notch filter at around $30 \mathrm{GHz}$ is experimentally demonstrated and $100 \%$ fractional tuning over $360^{\circ}$ range is achieved without changing the shape of the spectral response. The tuning mechanism is based on the use of slow and fast light effects in semiconductor optical amplifiers assisted by optical filtering.
\end{abstract}

Index Terms-Coherent population oscillations (CPO), microwave photonics, semiconductor optical amplifier (SOA), slow light.

\section{INTRODUCTION}

M ICROWAVE photonics has already attracted considerable interest for processing microwave or millimeter-wave signals directly in the optical domain [1]-[3], due to its huge bandwidth, inherent speed, immunity to electromagnetic interference, and low loss. One important application of interest is the microwave photonic notch filter, which has potential for broadband wireless access networks and radar systems. To satisfy the expected bandwidth demand in the coming years, several millimeter-wave frequency bands, such as $\sim 28$ and $\sim 40 \mathrm{GHz}$, which can provide transfer rates higher than $2 \mathrm{MHz}$, have already been allocated for these systems [4]. During the past several decades, many approaches to realize tunable notch filters have been proposed [5], e.g., liquid crystal spatial light modulator [6], fiber Bragg gratings (FBGs) [7], arrayed waveguide gratings [8], and optical attenuators [9]. However, all the above techniques result in a simultaneous undesirable variation of the free spectral range (FSR) while the filters are continuously tuned. In [10] and [11], it has been shown that this undesirable effect can be avoided by using microwave photonic phase shifters capable of providing a tunable phase-shift over the full $360^{\circ}$ range.

On the other hand, the use of slow and fast light effects based on the physical phenomena of electromagnetically induced transparency and coherent population oscillations (CPO) [12],

Manuscript received October 02, 2008; revised November 03, 2008. First published November 21, 2008; current version published January 16, 2009. This work was supported by the Danish Research Council project QUEST, by the European Union Network of Excellence ePIXnet, by the European Union FP7 projects GOSPEL and ACCORD, and by the Plan Nacional I+ D TEC200768065-C03-01.

W. Xue and J. Mørk are with DTU Fotonik, Department of Photonics Engineering, Technical University of Denmark, 2800 Kgs. Lyngby, Denmark (e-mail: wexu@fotonik.dtu.dk; jesm@ fotonik.dtu.dk).

S. Sales and J. Capmany are with the ITEAM Institute, Universidad Politecnica de Valencia, 46022 Valencia, Spain (e-mail: ssales@gco.upv.es; jcapmany@dcom.upv.es).

Color versions of one or more of the figures in this letter are available online at http://ieeexplore.ieee.org.

Digital Object Identifier 10.1109/LPT.2008.2009468
[13], to achieve a microwave phase shifter [14]-[18], has received considerable attention lately. In particular, semiconductor-based devices provide advantages such as the possibility of integration, low power consumption, and operation at room temperature. Many results of controlling the microwave phase of sine-modulated optical signals have been demonstrated [16]-[19], which include a $\sim 120^{\circ}$ phase shift at the modulation frequency of $4 \mathrm{GHz}$ in two semiconductor optical amplifier-electroabsorber (SOA-EA) pairs [17] and $\sim 200^{\circ}$ phase shift at $1 \mathrm{GHz}$ in a $2.5-\mathrm{mm}$ quantum-well SOA [18]. In [19], we reported that optical filtering can exploit the refractive-index dynamics of the SOA to not only largely enhance the phase slow-down effect, but also greatly increase the available bandwidth from a few to tens of gigahertz. Furthermore, by controlling the optical phase difference between the carrier and sidebands of the input optical signal, both $\sim 120^{\circ}$ phase delay and $\sim 170^{\circ}$ phase advance at $19 \mathrm{GHz}$ can be realized by slow and fast light effects in an SOA assisted by optical filtering [20], [21].

In this letter, we demonstrate for the first time, to the best of our knowledge, $100 \%$ fractional tuning over $360^{\circ}$ range of a microwave photonic notch filter based on slow and fast light effects in an SOA.

\section{PRINCIPLES OF OPERATION}

\section{A. Microwave Phase Shifter Based on Slow and Fast Light Effects in SOAs}

In [19], we have already demonstrated that, for a sinusoidally modulated optical signal at microwave frequency $\Omega$, employing an FBG notch filter to suppress the red-shifted sideband after the SOA and before the photodetection can greatly enhance the phase change induced by $\mathrm{CPO}$ effects. $\mathrm{A} \sim 150^{\circ}$ phase delay at the modulation frequency of $19 \mathrm{GHz}$ has been observed and is found to be due to the refractive-index dynamics in the active region of the device. But this is still not enough to achieve a shift of the null frequency of a notch filter by an amount given by the FSR. In [20] and [21], based on a similar setup as used in [19], both $\sim 120^{\circ}$ phase delay and $\sim 170^{\circ}$ phase advance have been successfully realized by choosing different values for the initial optical phase difference between the carrier and sidebands, which provides a possible way to achieve a $\sim 360^{\circ}$ phase shifter.

Fig. 1 shows the basic architecture of the microwave phase shifter. The detailed method to measure the phase shift is similar to that described in [14] and [19]. The RF intensity modulation is imposed on a continuous-wave laser beam using a Mach-Zehnder modulator (MZM). By operating the MZM at the different transmission slopes, $V_{1}$ and $V_{2}, \sim 0^{\circ}$ or $\sim 180^{\circ}$ 


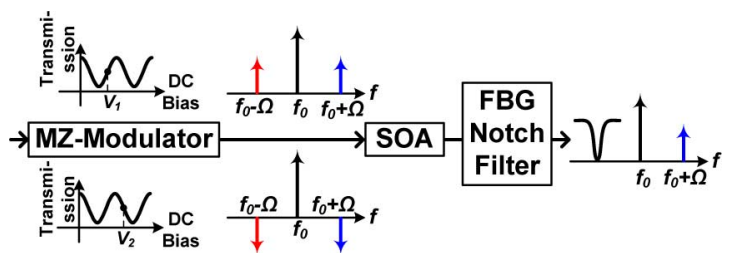

Fig. 1. Schematic of the microwave photonic phase shifter. $\left(f_{0}\right.$ : laser frequency; $\Omega$ : microwave modulation frequency; $V_{1}$ and $V_{2}$ : DC bias voltages of the modulator).

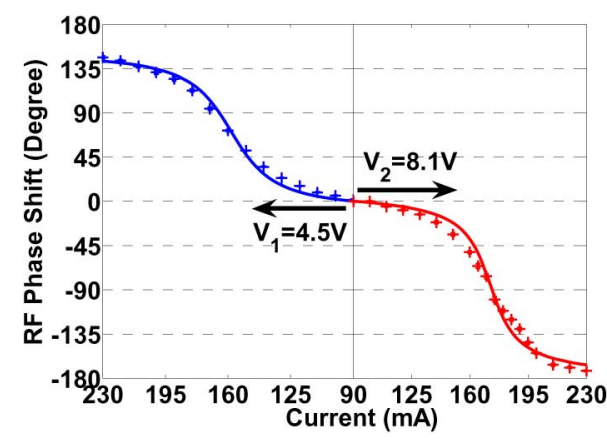

Fig. 2. Phase shifts as a function of injection current of the SOA when the modulator is biased at $V_{1}=4.5 \mathrm{~V}$ (blue curves in the left part) and $V_{2}=8.1 \mathrm{~V}$ (red curves in the right part). The " + " markers show experimental results and the solid lines are numerical simulations. (The modulation frequency is sweeping between 29.75 and $30.25 \mathrm{GHz}$.)

phase difference between the carrier and sidebands can be obtained.

Fig. 2 illustrates the phase shifts induced by slow and fast light effects through changing the injection current of the SOA for two different operating bias voltages of the modulator. For these two cases, the reference phase is chosen at the injection current of $90 \mathrm{~mA}$, and the input optical power into the SOA is fixed at $9 \mathrm{dBm}$. The laser wavelength is $1552.7 \mathrm{~nm}$. When the MZM is operated at the positive slope $\left(V_{1}=4.5 \mathrm{~V}\right)$, about $150^{\circ}$ phase delay at $30 \mathrm{GHz}$ is achieved, as shown in the left part of Fig. 2. On the other hand, about $170^{\circ}$ phase advance, shown in the right part of Fig. 2, is obtained for the negative slope $\left(V_{2}=8.1 \mathrm{~V}\right)$. Fig. 2 also shows that the phase sensitivity is different over the whole tuning range, $\sim 12^{\circ}$ phase shift due to a 5-mA current change at the injection current of around $170 \mathrm{~mA}$ and $\sim 2^{\circ}$ phase shift due to the same current change at around 90 or $230 \mathrm{~mA}$. The solid lines are numerical results, calculated using a four-wave mixing model appropriate for semiconductor waveguides [14], [18], and show good agreement with the experimental results. In [19], we have already shown that the optical filtering technique can increase the bandwidth of the phase shifter to at least $15 \mathrm{GHz}$, which means that this proposed microwave phase shifter can provide $\sim 360^{\circ}$ phase shift at other microwave frequency bands. The lower microwave frequency is limited to several gigahertz by the bandwidth of the optical FBG notch filter, which is employed to block the red-shifted sideband.

\section{B. Experimental Setup}

Fig. 3 describes the experimental scheme. The filter itself is a simple Mach-Zehnder interferometer composed of two arms, one of which incorporates the microwave phase shifter, shown

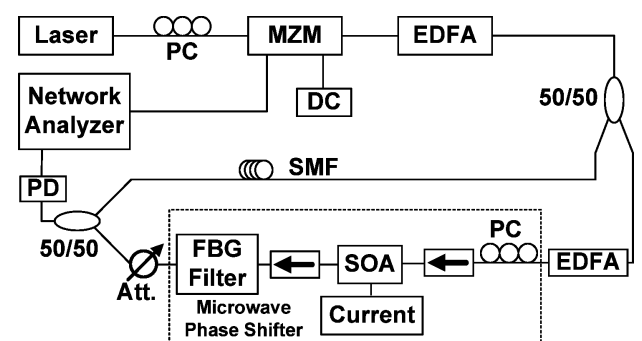

Fig. 3. Experimental setup of a tunable two-tap microwave photonic notch filter. (Att.: tunable attenuator; PC: polarization controller.)

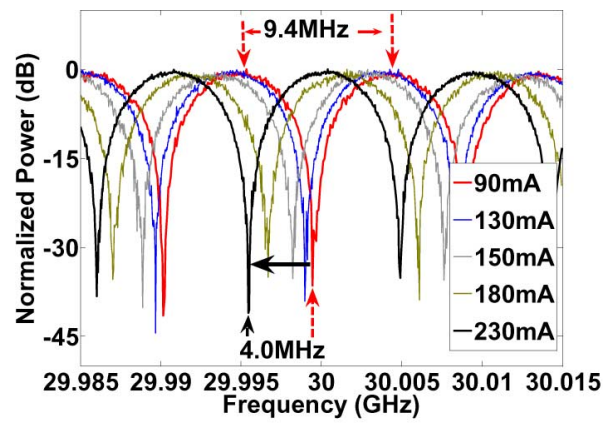

(a)

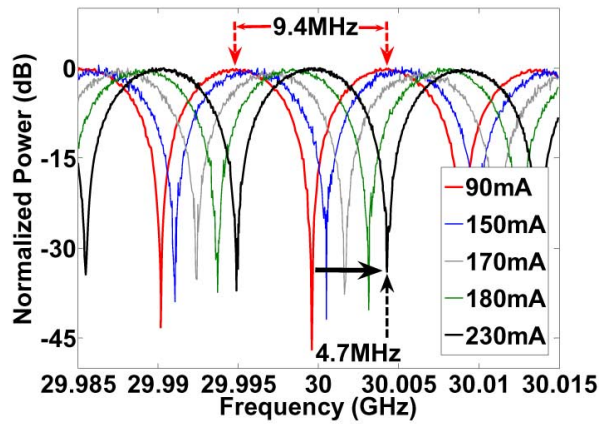

(b)

Fig. 4. Tunable RF response of the notch filter obtained by changing the injection current of the SOA, when the modulator is biased at (a) $V_{1}=4.5 \mathrm{~V}$ and (b) $V_{2}=8.1 \mathrm{~V}$.

in the dotted-line box, which is made up of an SOA followed by an FBG notch filter. The erbium-doped fiber amplifier is used to adjust the SOA input optical power to $9 \mathrm{dBm}$, in order to ensure that the SOA operates in the saturation regime. After the microwave phase shifter, a tunable attenuator provides amplitude balance between the two arms to compensate $\sim 10$-dB power change of the output signal after the SOA [19].

Tuning of the null frequency can be achieved by changing the injection current of the SOA. Through switching between the two operating stages $\left(V_{1}=4.5 \mathrm{~V}\right.$ and $\left.V_{2}=8.1 \mathrm{~V}\right)$ of the MZM, a $330^{\circ}$ phase shift, which is the sum of an $\sim 150^{\circ}$ phase delay and $\sim 170^{\circ}$ phase advance, is expected. Note that the switching between the two different operating points of the MZM will not change the spectral shape of the filter.

\section{EXPERIMENTAL RESULTS}

Fig. 4 shows the measured filter responses for different currents. The FSR is $9.4 \mathrm{MHz}$, corresponding to $22-\mathrm{m}$ optical fiber length difference between the two arms. The notch rejection is 


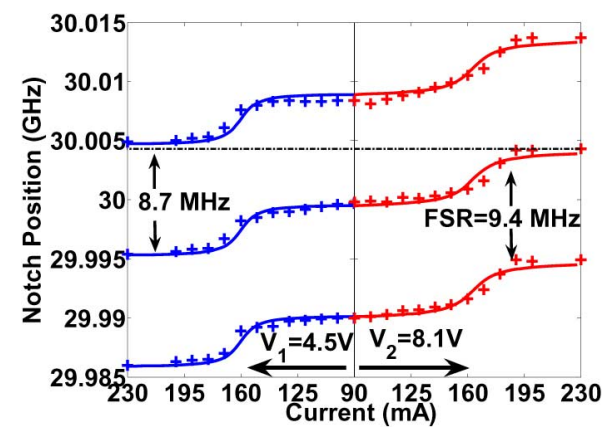

Fig. 5. Measured notch frequency versus injection current of the SOA, when the modulator is biased at $V_{1}=4.5 \mathrm{~V}$, (the blue curves in the left part) and $V_{2}=8.1 \mathrm{~V}$ (the red curves in the right part). The "+ " markers and the solid lines are experimental and numerical results, respectively.

always larger than $30 \mathrm{~dB}$ over the entire tuning range. The spectral response at the SOA injection current of $90 \mathrm{~mA}$ is chosen as the reference for both operating stages of the MZM.

For $V_{1}=4.5 \mathrm{~V}$, as shown in Fig. 4(a), the null frequency experiences a shift of $4.0 \mathrm{MHz}$ towards lower frequencies as the current increases from 90 to $230 \mathrm{~mA}$, which corresponds to almost $+50 \%$ fractional tuning range. Similarly, Fig. 4(b) shows that if the MZM is operated at the negative slope $V_{2}=8.1 \mathrm{~V}$, a 4.7-MHz shift in the other direction, corresponding to the $-50 \%$ fractional tuning range, is obtained by changing the current from 90 to $230 \mathrm{~mA}$. Note that the two initial frequency responses at $90 \mathrm{~mA}$ in Fig. 4(a) and (b) have the same position as well as the same shape. Fig. 5 demonstrates that through switching between the two operation points of the MZM, the notch positions of the microwave photonic notch filter can be continuously tuned over 8.7 MHz, without changing the spectral shape of the response, by changing the injection current of the SOA. The solid lines are simulation results. Because the phase shifts shown in Fig. 2 are measured over a bandwidth of $500 \mathrm{MHz}$, this almost $100 \%$ fractional tuning range will be maintained for even larger filter FSRs, which can be achieved by decreasing the time difference between two arms. Notice, however, that for the time difference on the order of or smaller than the coherence time of the laser, optical interference effects have to be taken into account [5].

\section{CONCLUSION AND OUTLOOK}

We have presented a novel scheme to achieve a widely tunable microwave photonic notch filter, which is based on slow and fast light effects in SOAs and can realize 100\% fractional tuning range at $30 \mathrm{GHz}$. The experimental results agree well with numerical results and show the potential for the application of this technique in the new generation of mobile broadband systems operating in the millimeter-wave frequency range. The advantages of the proposed scheme are large tuning range, wide choice of frequency bands, low control voltage, and fast reconfiguration in the order of a few nanoseconds or several hundreds of picoseconds, which is only determined by the switching time of the SOA because the switching of the bias points of the MZM could be avoided by using a specially designed MZM. Furthermore, the tunable attenuator used to balance the power between the two arms could be replaced by an EA, rendering the entire setup promising for future integrated devices.

\section{REFERENCES}

[1] J. Capmany and D. Novak, "Microwave photonics combines two worlds," Nature Photon., vol. 1, no. 6, pp. 319-330, Jun. 2007.

[2] A. J. Seeds and K. J. Williams, "Microwave photonics," J. Lightw. Technol., vol. 24, no. 12, pp. 4628-4641, Dec. 2006.

[3] R. A. Minasian, "Photonic signal processing of microwave signals," IEEE Trans. Microw. Theory Tech., vol. 54, no. 2, pt. 2, pp. 832-846, Feb. 2006.

[4] IEEE Standard for Local and Metropolitan Area Networks, Part 16: Air Interface for Fixed and Mobile Broadband Wireless Access Systems, IEEE Standard 802.16, 2006

[5] J. Capmany, B. Ortega, D. Pastor, and S. Sales, "Discrete-time optical processing of microwave signals," J. Lightw. Technol., vol. 23, no. 2, pp. 702-723, Feb. 2005.

[6] D. Dolfi, J. Tabourel, O. Durand, V. Laude, and J. Huignard, "Optical architectures for programmable filtering and correlation of microwave signals," IEEE Trans. Microw. Theory Tech., vol. 45, no. 8, pp. 1467-1472, Aug. 1997

[7] D. Pastor, J. Capmany, and B. Ortega, "Broadband tunable microwave transversal notch filter based on tunable uniform fiber Bragg gratings as slicing filters," IEEE Photon. Technol. Lett., vol. 13, no. 7, pp. 726-728, Jul. 2001.

[8] F. Coppinger, S. Yegnanarayanan, P. D. Trinh, B. Jalali, and I. L. Newberg, "Nonrecursive tunable photonic filter using wavelength-selective true time delay," IEEE Photon. Technol. Lett., vol. 8, no. 9, pp. 1214-1216, Sep. 1996.

[9] N. You and R. A. Minasian, "A novel tunable microwave optical notch filter," IEEE Trans. Microw. Theory Tech., vol. 49, no. 10, pp. 2002-2005, Oct. 2001.

[10] F. Coppinger, S. Yegnanarayanan, P. D. Trinh, and B. Jalali, "Continuously tunable photonic radio-frequency notch filter," IEEE Photon. Technol. Lett., vol. 9, no. 3, pp. 339-341, Mar. 1997.

[11] A. Loayssa, J. Capmany, M. Sagues, and J. Mora, "Demonstration of incoherent microwave photonic filters with complex coefficients," IEEE Photon. Technol. Lett., vol. 18, no. 16, pp. 1744-1746, Aug. 15, 2006.

[12] L. V. Hau, S. E. Harris, Z. Dutton, and C. H. Behroozi, "Light speed reduction to 17 meters per second in an ultracold atomic gas," Nature, vol. 397, pp. 594-598, Feb. 1999.

[13] M. S. Bigelow, N. N. Lepeshkin, and R. W. Boyd, "Superluminal and slow light propagation in a room-temperature solid," Science, vol. 301, pp. 200-202, Jul. 2003

[14] J. Mørk, R. Kjær, M. Van der Poel, and K. Yvind, "Slow light in a semiconductor waveguide at gigahertz frequencies," Opt. Express, vol. 13, no. 20, pp. 8136-8145, Oct. 2005.

[15] C. J. Chang-Hasnain and S. L. Chuang, "Slow and fast light in semiconductor quantum-well and quantum-dot devices," J. Lightw. Technol., vol. 24, no. 12, pp. 4642-4654, Dec. 2006.

[16] S. Sales, F. Öhman, J. Capmany, and J. Mørk, "Controlling microwave signals by means of slow and fast light effects in SOA-EA structures," IEEE Photon. Technol. Lett., vol. 19, no. 20, pp. 1589-1591, Oct. 15, 2007.

[17] F. Öhman, K. Yvind, and J. Mørk, "Slow light in a semiconductor waveguide for true-time delay applications in microwave photonics," IEEE Photon. Technol. Lett., vol. 19, no. 15, pp. 1145-1147, Aug. 1, 2007.

[18] P. K. Kondratko and S. L. Chuang, "Slow-to-fast light using absorption to gain switching in quantum-well semiconductor optical amplifier," Opt. Express, vol. 15, no. 16, pp. 9963-9969, Aug. 2007.

[19] W. Xue, Y. Chen, F. Öhman, S. Sales, and J. Mørk, "Enhancing light slow-down in semiconductor optical amplifiers by optical filtering," Opt. Lett., vol. 33, no. 10, pp. 1084-1086, May 2008.

[20] W. Xue, Y. Chen, F. Öhman, S. Sales, and J. Mørk, "Chirp dependence of filter assisted slow and fast light effects in semiconductor optical amplifiers," in OSA Topical Meeting Slow and Fast Light, Boston, MA, Jul. 13-16, 2008, Paper JMB12.

[21] W. Xue, Y. Chen, F. Öhman, and J. Mørk, "The role of input chirp on phase shifter based on slow and fast light effects in semiconductor optical amplifiers," Opt. Express, submitted for publication. 